\title{
Psychologie ergonomique du travail collectif assisté par ordinateur : l'utilisation du collecticiel dans les projets de conception de produits
} Résumé de thèse en Psychologie soutenue le 20 novembre 2006

\section{Guillaume Gronier}

\section{OpenEdition}

Journals

Édition électronique

URL : http://journals.openedition.org/activites/1976

DOI : 10.4000/activites. 1976

ISSN : $1765-2723$

Éditeur

ARPACT - Association Recherches et Pratiques sur les ACTivités

\section{Référence électronique}

Guillaume Gronier, «Psychologie ergonomique du travail collectif assisté par ordinateur : I'utilisation du collecticiel dans les projets de conception de produits », Activités [En ligne], 5-1 | avril 2008, mis en ligne le 15 avril 2008, consulté le 15 septembre 2020. URL : http://journals.openedition.org/activites/ 1976

\section{(c) (†) $\odot$}

Activités est mis à disposition selon les termes de la licence Creative Commons Attribution - Pas d'Utilisation Commerciale - Pas de Modification 4.0 International 


\title{
Psychologie ergonomique du travail collectif assisté par ordinateur : \\ l'utilisation du collecticiel dans les projets de conception de produits
}

\author{
Soutenue le 20 novembre 2006 par
}

\section{Guillaume Gronier}

Jury de thèse: Nathalie Bonnardel (rapporteur); Pierre Falzon (président du jury); Cécile van de Leemput (rapporteur); Jean-Pierre Minary (codirecteur de thèse); Jean-Claude Sagot (directeur de thèse)

\section{Mots clés}

Coopération, coordination, communication, travail collectif, conception de produits, collecticiel, travail coopératif assisté par ordinateur.

\section{1.- De nouveaux modèles d'organisation du travail}

Le contexte socio-économique à forte concurrence, perpétuellement en évolution, est le plus souvent responsable des transformations technologiques et organisationnelles qui s'opèrent dans les structures de production. Ces transformations passent non seulement par le réaménagement des méthodes de conception, mais aussi, et surtout, par l'étroite collaboration des différents métiers (ingénieurs, designers, ergonomes, etc.) travaillant sur un même projet.

Dès lors, les outils de Travail Coopératif Assisté par Ordinateur (TCAO), aussi appelés collecticiels, prennent une place importante au sein des nouvelles organisations car ils permettent de structurer les activités collectives à travers l'échange et le partage de documents, la coordination des actions coopératives, la gestion des communications synchrones et asynchrones ou encore l'instrumentation des prises de décisions collaboratives. Néanmoins, la réelle influence de ces outils sur les activités collectives des équipes de projet pluridisciplinaires en conception de produits est encore mal connue.

\section{2.- Une approche du travail collectif}

En accord avec Soubie, Buratto et Chabaud (1996), nous considérons le travail collectif comme le rapport entre les activités de coopération, de coordination et de communication.

Pour ces auteurs, la coopération est le processus de raisonnements et/ou de mise en commun de connaissances dans le cadre de la résolution de problèmes. En conception de produits, la coopération implique des phases de travail conjointes (co-conception) ou séparées (conception distribuée) (Darses, \& Falzon, 1996).

La coordination apparaît comme le complément de l'activité de coopération. Maggi (1997) précise que la coordination n'est que l'ensemble des règles d'action qui structure la coopération. Jeantet (1998) ajoute que la coordination est assurée par les objets intermédiaires de conception (documents, maquettes, esquisses, etc.) qui sont au cœur des interactions interindividuelles.

Pour finir, la communication est souvent décrite comme indispensable à la dimension collective du 
travail. Plus précisément, ce sont les communications verbales qui sont privilégiées dans le cadre de l'étude du travail collectif.

Le travail collectif à travers les réseaux informatiques est assuré par les collecticiels, définis par Favier (1998) comme « l'ensemble des techniques et des méthodes qui contribuent à la réalisation d'un objectif commun à plusieurs acteurs, séparés ou réunis par le temps et l'espace, à l'aide de tout dispositif interactif faisant appel à l'informatique, aux télécommunications et aux méthodes de conduite de groupe ». Les collecticiels sont ainsi considérés comme des outils pour l'interaction, privilégiant la communication (messagerie et forum électroniques), la coopération (partage et réplication d'informations) et la coordination (gestion du temps, gestion des tâches, échanges de documents).

\section{3.- Problématique générale : vers un travail collectif assisté par ordinateur en conception}

La problématique de cette thèse s'inscrit au cœur des recherches menées en psychologie ergonomique sur le travail collectif assisté par ordinateur. Il s'agit d'identifier les effets de la médiation sur les équipes de travail, pour en déterminer les avantages, les contraintes et les complémentarités par rapport aux situations de travail collectif plus traditionnelles, c'est-à-dire généralement organisées sous la forme de réunions de projet en présence.

Du point de vue méthodologique, l'utilisation libre d'un collecticiel, baptisé Atelier Coopératif de Suivi de Projet (ACSP) (Gomes, \& Sagot, 2003), est observée au contact de plusieurs situations de travail. Ce collecticiel est mis en œuvre pour recueillir des données issues du terrain, en accord avec une démarche naturaliste (observation avec instruments et analyses des traces) largement reconnue en psychologie ergonomique.

\section{4.- Situations d'analyse du travail collectif}

Trois situations de travail collectif ont été retenues et font l'objet d'études complémentaires. Elles permettent ainsi d'aborder le travail collectif depuis ses aspects organisationnels, en décrivant le cadre général de la gestion de projets, jusqu'à ses aspects individuels, à travers une approche sociocognitive de l'activité de conception puis par l'analyse d'une situation de coopération multisites.

\section{1.- Appropriation du collecticiel pour la gestion de projets}

La première situation étudiée porte sur la gestion de projets conduits par une organisation. L'appropriation du collecticiel par les utilisateurs y est analysée, en tenant compte des facteurs de résistance au changement et des modifications opératoires qui sont induites par le système collaboratif. Méthodologiquement, nous nous sommes appuyés sur l'analyse des traces informatiques (connexions au collecticiel et pages consultées), ainsi que sur différents entretiens semi-directifs réalisés auprès des acteurs.

L'enregistrement des projets au sein de l'ACSP a montré que le système organisationnel était structuré selon le modèle de l'organisation-projet-réseau, c'est-à-dire qu'il regroupait un ensemble de projets gérés de façon autonome, mais interdépendants du point de vue des ressources humaines et matérielles. Dans le cadre de l'utilisation de l'ACSP, nous avons observé trois étapes principales qui marquent l'appropriation progressive du collecticiel, accompagnée d'une période de résistance au changement puis de déplacement.

\section{2.- Un projet de conception multimétiers}

La deuxième situation est centrée sur la coopération inter-métiers au cours d'un grand projet industriel de conception de produits impliquant des ingénieurs et des ergonomes. Dans une approche sociocognitive, l'analyse porte sur l'implication des acteurs-métiers selon le contexte de coopération 
(en présence ou assisté par ordinateur) et selon les phases du processus de conception. Les traces informatiques (connexions et pages consultées) et l'analyse des échanges conversationnels au cours de 3 réunions de projet par l'intermédiaire du logiciel ALCESTE (Reinert, 2001) ont permis de dégager plusieurs résultats.

De point de vue des métiers, l'ergonome tient le rôle de médiateur: il sert la conception du produit par la définition de concepts en orientant les réflexions du groupe vers de nouvelles représentations centrées sur l'activité future souhaitable (Sagot, \& Gomes, 2003). L'ingénieur, quant à lui, tient le rôle d'acteur: il sert le développement du produit à travers la proposition de solutions techniques.

Du point de vue technologique, le collecticiel offre un espace de travail partagé de type plateau-projet (Levan, 2004) favorable à la coopération. Toutefois, les échanges médiatisés orientent le travail collectif vers le développement du produit au détriment de la définition du concept. En ce sens, le collecticiel offre un support plus favorable au métier d'ingénieur qu'au métier d'ergonome.

\section{3.- Travail collectif à distance}

À partir d'un protocole expérimental, la troisième situation compare les activités collectives d'une équipe projet coopérant à distance par rapport à une autre équipe projet coopérant en présence. Des statistiques issues des traces informatiques (utilisation du collecticiel par les acteurs des projets), ainsi que l'analyse par le logiciel ALCESTE des échanges conversationnels au cours des réunions de projet et des contenus des messages écrits (courriels et messages du forum) enregistrés dans l'ACSP, nous ont permis d'étudier l'activité selon chaque contexte de coopération.

Les résultats montrent notamment que le collecticiel est largement utilisé pour la capitalisation de données et l'élaboration d'une mémoire organisationnelle. De plus, l'outil collaboratif favorise une démarche d'ingénierie concourante en structurant les activités de coordination. On observe toutefois certaines inégalités face à l'appropriation de la technologie par les acteurs de la conception.

\section{5.- Conclusions et perspectives de recherche}

S'il existe une réelle volonté de concilier les thématiques des nouvelles technologies, du travail collectif et de l'activité de conception, peu de travaux sont encore menés en conciliant ces trois aspects. Ceci peut s'expliquer par le caractère très récent des technologies collaboratives appliquées au processus de conception. En 1997, Cardon soulignait « la diffusion extrêmement faible des machines à coopérer », qui interdisait « d'entreprendre de grandes enquêtes sur les usagers participant à des collectifs de travail médiatisé par des produits de CSCW ». Un peu plus tard, Olson et Olson (2002) constataient une large diffusion de collecticiels commerciaux, mais indiquaient que des recherches scientifiques menées sur les effets organisationnels et les usages individuels des collecticiels étaient encore à réaliser. En 2007, la situation a évolué timidement. Car si l'on comptabilise désormais un grand nombre de collecticiels, la plupart restent encore le produit de laboratoires de recherche ou prennent place sur le terrain avec beaucoup de réserve.

Par ailleurs, sur la base de notre travail, plusieurs pistes peuvent être dégagées pour le développement des collecticiels dédiés à la conception de produits. Ainsi, il paraît important d'orienter l'architecture des outils collaboratifs vers le management et la capitalisation des connaissances, en structurant toutes les données échangées selon un canevas défini par les besoins de l'entreprise. L'organisation temporelle ou thématique de ces données ne saurait suffire à restituer de façon efficace les traces des décisions prises au cours du processus de conception.

De plus, l'espace de travail partagé doit fournir une visibilité sur la contribution de chacun, afin d'engager une dynamique de groupe autour du projet. Des indicateurs simples, comme les dernières visites de chaque membre, pourraient favoriser la conscience mutuelle et l'élan de collaboration. 


\section{BIBLIOGRAPHIE REPRÉSENTATIVE}

Cardon, D. (1997). Les sciences sociales et les machines à coopérer: une approche bibliographique du Computer Supported Cooperative Work. Réseaux, 85, 13-51.

Darses, F., \& Falzon, P. (1996). La conception collective: une approche de l'ergonomie cognitive. In G. de Terssac, \& E. Friedberg (Eds.), Coopération et Conception (pp. 123-135). Toulouse: Octarès Editions.

Favier, M. (1998). (Ed.). Le travail en groupe à l'âge des réseaux. Paris: Economica.

Gomes, S., \& Sagot, J.C. (2003). A concurrent engineering experience based on a cooperative and object oriented design methodology. In P. Chedmail, G. Cognet, C. Fortin, C. Mascle, \& J. Pegna (Eds.), Integrating design and manufacturing in mechanical engineering (pp. 11-18). London: Kluwer Academic Publishers.

Jeantet, A. (1998). Les objets intermédiaires dans la conception. Eléments pour une sociologie des processus de conception. Sociologie du travail, 3, 291-316.

Levan, S.K. (2004). Travail collaboratif sur Internet. Concepts, méthodes et pratiques des plateaux projet. Paris: Vuibert.

Maggi, B. (1997). Coopération et coordination dans et pour l'ergonomie: quelques repères. Performances Humaines et Techniques, Hors série: Coopérations et coordinations dans l'intervention en ergonomie. Frontières, multidisciplinarité, collectifs d'action, 11-15.

Olson, G.M., \& Olson, J.S. (2002). Groupware and computer-supported cooperative work. In J.A. Jacko, \& A. Sears (Eds.), The human-computer interaction handbook (pp. 583-595). Mahwah: Lawrence Erlbaum Associates.

Reinert, M. (2001). Alceste, une méthode statistique et sémiotique d'analyse de discours; application aux «Rêveries du promeneur solitaire ». La Revue Française de Psychiatrie et de Psychologie Médicale, 49 (5), 32-46.

Sagot, J.C., \& Gomes, S. (2003). Intégration des facteurs humains dans la démarche de conception. Une approche ergonomique. Cahiers de notes documentaires - Hygiène et sécurité du travail, 191, 61-71.

Soubie, J.L., Buratto, F., \& Chabaud, C. (1996). La conception de la coopération et la coopération dans la conception. In G. de Terssac \& E. Friedberg (Eds.), Coopération et conception (pp. 187-206). Toulouse: Octarès Editions. 